\title{
Anestesiologia Baseada em Evidências: O Que É e Como Praticar *
}

\section{Evidence-Based Anesthesiology: What Is It and How to Practice It}

Rodrigo Mariano da Costa de Angelis, TSA ${ }^{1}$; Álvaro Avezum Júnior ${ }^{2}$; Alexandre Biasi Cavalcanti ${ }^{3}$; Rogério Teixeira de Carvalho ${ }^{4}$

\section{RESUMO}

Angelis RMC, Avezum Jr A, Cavalcanti AB, Carvalho RT - Anestesiologia Baseada em Evidências: O Que É e Como Praticar

JUSTIFICATIVA E OBJETIVOS: Os princípios da Medicina baseada em evidências têm sido aplicados à Anestesiologia. A proposta dessa revisão é abordar os fundamentos para a prática da Medicina baseada em evidências e fornecer exemplos de como esses princípios podem ser incorporados à prática diária. Até o momento da conclusão desse artigo, não foi encontrado nenhum trabalho sobre Anestesiologia baseada em evidências na literatura médica brasileira, o que determinou a sua realização.

CONTEÚDO: Este artigo consiste de revisão que aborda um novo paradigma da Medicina e como ele pode ser aplicado à Anestesiologia.

CONCLUSÕES: A Anestesiologia baseada em evidências constitui a integração da melhor evidência científica disponível à experiência clínica do anestesiologista e às expectativas do paciente, visando à melhoria do cuidado com ele.

Unitermos: ANESTESIOLOGIA: anestesia baseada em evidências; EPIDEMIOLOGIA, Clínica

\section{SUMMARY}

Angelis RMC, Avezum Jr A, Cavalcanti AB, Carvalho RT - Evidence-Based Anesthesiology: What Is It and How to Practice It

BACKGROUND AND OBJECTIVES: The principles of evidence-based medicine have been applied to Anesthesiology. The purpose of this article is to review the basis for the practice of evidence-based medicine and give some examples of how its principles may be incorporated to the clinical practice. We couldn't find any article about this issue in the Brazilian medical literature and decided to perform it.

CONTENTS: This study is a review focusing on a new medical paradigm and how it can be applied to Anesthesiology.

CONCLUSIONS: Evidence-Based Anesthesiology is the integration of best scientific evidence, anesthesiologists' clinical experience and patients'expectations, aiming at improving patients'care.

Key Words: ANESTHESIOLOGY: evidence-based anesthesia; EPIDEMIOLOGY, Clinical

\section{INTRODUÇÃO}

$\mathrm{M}$ edicina baseada em evidências, expressão utilizada no Brasil pela primeira vez por Avezum ${ }^{1}$ e cuja origem filosófica remonta à França, século XIX, constitui a utilização conscienciosa, explícita e judiciosa da melhor evidência visando à tomada de decisão para o tratamento individual dos pacientes ${ }^{2}$. O princípio fundamental da Medicina baseada em evidências é o uso efetivo da literatura médica como guia para a prática diária ${ }^{3}$. Três são seus

\footnotetext{
Recebido do (Received from) Instituto de Ensino e Pesquisa do Hospital Israelita Albert Einstein, São Paulo, SP

1. Anestesiologista do Hospital Israelita Albert Einstein, São Paulo, SP

2. Coordenador Executivo do Centro Brasileiro de Estudos Clínicos - Instituto Dante Pazzanese de Cardiologia; Coordenador de Centro de Pesquisa Clínica do Instituto de Ensino e Pesquisa do Hospital Israelita Albert Einstein, São Paulo, SP

3. Especialista em Cardiologia

4. Membro da Sociedade Brasileira de Ortopedia, Membro da Sociedade Brasileira de Cirurgia do Joelho
}

Apresentado (Submitted) em 14 de agosto de 2003 Aceito (Accepted) para publicação em 06 de novembro de 2003

Endereço para correspondência (Correspondence to)

Dr. Rodrigo Mariano da Costa de Angelis

Rua José Maria Lisboa, 155/84

01423-000 São Paulo, SP

E-mail: rodrigomariano@terra.com.br

(C) Sociedade Brasileira de Anestesiologia, 2004 elementos-chave: a melhor evidência científica, a experiência clínica do médico e as preferências do paciente.

A expressão Medicina baseada em evidências surgiu na literatura médica há cerca de duas décadas ${ }^{4} \mathrm{e}$, apesar de inicialmente relacionada à prática da Medicina Interna, foi incorporada à Anestesiologia. O primeiro livro de Medicina baseada em evidências em Anestesiologia foi publicado em 2000, na Inglaterra ${ }^{5}$.

A última década testemunhou interesse crescente pelo assunto. Sackett e col. ${ }^{6}$ o atribuíram a quatro motivos básicos: a necessidade crescente de conhecimentos sobre diagnóstico, prognóstico, terapia e prevenção; limitações das fontes tradicionais de informação, tais como livros-texto (freqüentemente desatualizados), especialistas (muitas vezes emitindo opiniões incorretas e com fundamentação científica inadequada) e cursos de educação médica continuada (a maioria pouco efetivos); a disparidade entre a capacidade diagnóstica do médico e o seu raciocínio clínico, que costumam melhorar com a idade e com a experiência, e o seu conhecimento científico e desempenho clínico, que costumam diminuir com a idade e a escassez de tempo para o estudo, que resulta, na grande maioria dos casos, em apenas trinta minutos por semana de dedicação à procura e assimilação do conhecimento ${ }^{4}$.

Estima-se que sejam necessárias três horas e dezenove minutos diários de estudo para que um médico se mantenha atualizado dentro da sua especialidade. 
As Sociedades de Anestesiologia têm procurado aplicar os princípios da Medicina baseada em evidências para melhorar o cuidado dos pacientes. Como resultado, os anestesiologistas são considerados pioneiros no desenvolvimento e na adoção de diretrizes, que parecem ter contribuído para redução sustentada e disseminada da mortalidade e da morbidade relacionadas à anestesia ${ }^{7}$. Contudo, é difícil estabelecer uma relação causal forte entre a atividade dessas Sociedades e a diminuição da morbimortalidade.

\section{A PRÁTICA DA MEDICINA BASEADA EM EVIDÊNCIAS}

A prática da Medicina baseada em evidências significa a integração da experiência clínica individual à melhor evidência externa disponível, proveniente da pesquisa clínica ${ }^{8}$, e consiste em cinco passos básicos: converter a necessidade de informação em uma pergunta clínica bem formulada; procurar pela melhor evidência para responder à pergunta; avaliar a evidência quanto à sua validade e aplicabilidade à prática clínica; integrar a melhor evidência à experiência pessoal e às preferências do paciente, colocando-a em prática e avaliar a efetividade das medidas adotadas e procurar melhoria contínua $^{9}$.

Como pode a Medicina baseada em evidências ser aplicada à Anestesiologia? Ela pode ser incorporada à prática diária e também às reuniões de revista, nas discussões de casos clínicos e no ensino da especialidade. Os anestesiologistas podem trocar suas reuniões de revista e discussões de casos baseados em revisões não sistematizadas por revisões sistematizadas, com aporte em casos clínicos e com avaliação crítica da literatura.

\section{A FORMULAÇÃO DA PERGUNTA CLÍNICA}

A formulação de uma pergunta clínica bem elaborada constitui o passo inicial para a tomada de decisão em Medicina baseada em evidências. Aboa pergunta clínica deve ser estritamente relevante ao problema em questão e elaborada de tal forma a facilitar a busca pela resposta. Questões bem elaboradas têm quatro componentes-chave: (1) o paciente ou o problema de interesse; (2) a intervenção principal, ou seja, uma exposição, um teste diagnóstico, um fator prognóstico ou um regime de tratamento; (3) a comparação entre intervenções ou exposições, se for aplicável; (4) o desfecho clínico de interesse ${ }^{6}$.

As questões relacionadas à terapia, diagnóstico e prognóstico são de especial interesse para o anestesiologista. Alguns exemplos de questões clínicas em Anestesiologia podem ser enunciados: os pacientes submetidos à artroplastia total de quadril devem receber anestesia geral ou regional? Qual a melhor estratégia para prevenção de vômitos no período pós-operatório? Qual a probabilidade de um paciente com doença pulmonar restritiva, cuja capacidade vital pré-operatória eqüivale a $30 \%$ do normal, necessitar de ventilação mecânica no período pós-operatório? Em pacientes em que a exposição prévia à anestesia levantou a suspeita de hipertermia maligna, qual a capacidade do teste de contratura in vitro predizer quem poderá desenvolver o problema? O uso de heparina de baixo peso molecular em paciente do sexo feminino, de 80 anos de idade, submetida à cirurgia do quadril melhora seu prognóstico? Qual o risco de broncoespasmo num paciente que recebeu rapacurônio como bloqueador neuromuscular? ${ }^{10,11}$.

Após a elaboração de uma pergunta clínica bem estruturada, partiu-se em busca da melhor evidência disponível.

\section{A BUSCA DA MELHOR EVIDÊNCIA}

Existem, basicamente, quatro formas de se obter a informação: perguntar a alguém, consultar um livro-texto, encontrar um artigo pertinente ao assunto em seu arquivo pessoal ou utilizar uma base eletrônica de dados. Perguntar a um orientador ou a um colega é uma forma altamente eficiente; contudo, somente faz sentido quando se trata de um caso ou situação raros que o profissional dificilmente encontrará novamente. Consultar um livro-texto pode ser interessante, embora geralmente estejam desatualizados, desde o momento de sua publicação. Reportar-se a um artigo do arquivo pessoal incorre no risco de não terem sido mantidos os dados atualizados e completos.

Atualmente, as bases de dados eletrônicas constituem o meio mais rápido e atualizado de procurar informação. Amaior e mais utilizada fonte de dados utilizada é a MedLine, que conta com mais de 3500 revistas indexadas e contém cerca de 11 milhões de referências de 1966 até o presente. O tópico de assunto médico, MeSH (Medical Subject Heading), é o nome do vocabulário padrão utilizado para indexar as citações na MedLine. A organização hierárquica da base de dados obedece a uma estrutura ramificada que permite ao usuário utilizar termos mais específicos ou mais genéricos para mudar a estratégia de busca. A consulta ao MeSH permite a procura por tópicos, que provê um resultado melhor que a procura somente por palavras presentes no texto. A MedLine pode ser acessada através do PubMed da Biblioteca Nacional de Medicina (National Library of Medicine), pela Ovid Technologies, MDConsult e outras fontes eletrônicas de informação.

No caso específico da Anestesiologia, a informação está dispersa em periódicos de Medicina geral, de múltiplas sub-especialidades, incluindo a própria Anestesiologia, Pediatria e Cirurgia, além das ciências básicas.

A procura pela resposta na Medline deve ser baseada nos quatro elementos da pergunta clínica já citados.

A MedLine permite o acesso à maior parte da literatura biomédica publicada. Contudo, não avalia criticamente as referências contidas na base de dados. A análise da informação obtida constitui o passo seguinte para a prática da Medicina baseada em evidências. O médico que muito lê, mas não avalia a validade e a aplicabilidade da fonte, está bem informado, mas não aprendeu e não terá subsídios adequados para argumentar a favor de suas condutas. 


\section{A ANÁLISE DOS ESTUDOS}

\section{Estudos de Tratamento}

Para um estudo de tratamento, cinco perguntas devem ser realizadas ${ }^{12,13}$ : (1) Os resultados do estudo são válidos? (2) Quais foram os resultados obtidos? (3) Os benefícios do tratamento compensam os danos e os custos? (4) Os resultados ajudarão a melhorar a cuidar de meus pacientes? (5) Todos os desfechos clínicos importantes foram considerados?

\section{Os Resultados dos Estudos são Válidos?}

Primeiramente, devemos investigar se o estudo representa uma estimativa imparcial do efeito do tratamento em questão ou se há algum tipo de viés (bias), que significa a ocorrência de erro sistemático em um estudo epidemiológico que resulte em estimativa incorreta da associação entre exposição e desfecho. Essa questão pode ser respondida por meio das seguintes perguntas: (1) A seleção dos pacientes em cada grupo de tratamento foi realizada de forma aleatória? Adistribuição aleatória diminui o viés e assegura que os determinantes dos desfechos clínicos sejam distribuídos homogeneamente entre os grupos; (2) Todos os pacientes selecionados foram computados? Todos os pacientes que iniciaram o estudo o concluíram? Os pacientes que não concluíram o estudo foram incluídos na análise final? (3) Os pacientes foram analisados conforme os grupos para os quais foram alocados aleatoriamente no início do estudo (princípio da intenção de tratar)?; (4) Constitui um estudo duplamente encoberto? No estudo duplamente encoberto, o paciente não sabe qual opção de tratamento está recebendo e o investigador também não; (5) Com exceção da intervenção sendo investigada, os pacientes foram tratados de forma igual durante o estudo? Esta última questão está intimamente relacionada à anterior: nos estudos abertos, nos quais os médicos e os pacientes sabem que tratamento está sendo administrado, tanto a forma do paciente se comportar ao saber qual é o real tratamento que está recebendo, como a forma do clínico conduzir o caso pode ser modificada. O resultado final é que os resultados do estudo não refletirão apenas o efeito do tratamento em teste, mas também os efeitos de outros tratamentos que são diferentes entre os grupos. Este problema pode ser evitado, ao se adotar o esquema duplamente encoberto.

Um exemplo desse tipo de estudo que está relacionado à Anestesiologia é um estudo aleatório e duplamente encoberto comparando as drogas $\mathrm{A}$ e $\mathrm{B}$ quanto à eficácia na prevenção de vômitos no período peri-operatório, tal como o estudo realizado por Tang e col., que compararam a eficácia do ondansetron e do droperidol no tratamento da náusea no período peri-operatório de cirurgias ginecológicas eletivas ${ }^{14}$.

\section{Quais foram os Resultados?}

Duas perguntas devem ser realizadas em relação aos resultados: (1) Qual foi a magnitude do efeito terapêutico observado? (2) Qual a precisão da estimativa do efeito terapêutico?
Considere-se que no trabalho citado sobre prevenção de vômitos no período pós-operatório, entre os pacientes alocados para receber a droga $A$, a incidência de náuseas e vômitos tenha sido de $20 \%$, e que, no grupo alocado para a droga $\mathrm{B}$, o problema ocorreu em $15 \%$ dos casos. O risco de náuseas e vômitos pós-operatórios do grupo B em relação ao grupo Aé $0,15 / 0,20=0,75$. Esse é o chamado Risco Relativo (RR). O risco relativo é a razão da taxa de eventos no grupo tratado pela taxa de eventos no grupo controle (TET/TEC). Outro conceito importante é a redução do risco relativo (RRR), que constitui o valor da taxa de eventos no grupo controle menos a taxa de eventos no grupo tratado divido pela taxa de eventos no grupo controle (TEC-TET)/TEC ou (1-RR) x 100. A $R R R$ é expressa em porcentagem. A nova terapia empregada (droga $B$ ) reduziu o risco de náuseas e vômitos em $25 \%$ em relação à terapia convencional (droga $\mathrm{A}$ ). Mas, qual a precisão dessa redução de $25 \%$ ? Isso depende do número de pacientes envolvidos no estudo, ou seja, do tamanho da amostra. Admitindo que o trabalho inclua 100 pacientes; haveria 20 episódios de vômito no grupo $A$ e 15 no grupo $B$, uma diferença de apenas cinco episódios. Se o número de pacientes subisse para 1000; haveria 200 episódios no grupo $\mathrm{A}$ e 150 no grupo $B$, uma diferença de cinqüenta episódios. A RRR mantém-se a mesma, mas o intervalo de confiança será mais estreito, indicando que a precisão do resultado é maior. Isto ocorre porque o maior número de eventos diminui a possibilidade de que o valor encontrado tenha sido mera obra do acaso.

O intervalo de confiança informa os valores dentro dos quais o verdadeiro efeito deve estar situado. Combina informações sobre a força de uma associação (como a redução de risco relativo) com informações sobre os efeitos do acaso produzindo o resultado observado.

Por convenção, utiliza-se o intervalo de confiança de 95\%, que indica que há $95 \%$ de probabilidade de que o valor real da população esteja situado dentro do limite encontrado. Quanto maior for o tamanho da amostra, maior será o número de eventos observados e mais convencidos os leitores poderão ficar de que o resultado encontrado constitui uma representação verdadeira e mais precisa dos valores observados na população geral.

A interpretação dos intervalos de confiança é relativamente simples. Nos exemplos mencionados, há duas situações. No trabalho envolvendo 100 pacientes, o intervalo de confiança encontrado para RRR foi - $38 \%$ (um aumento da incidência de vômitos causado pela droga B) a 59\% (uma redução da incidência de vômitos pela droga B). Como o intervalo de confiança $95 \%$ inclui o zero, considera-se que o efeito estudado nãoé estatisticamente significante. No estudo envolvendo 1000 pacientes, o intervalo de confiança encontrado foi de $9 \%$ a $41 \%$. Isso significa que o leitor pode ter $95 \%$ de certeza de que a redução de vômitos com a droga $\mathrm{B}$, comparada à droga $\mathrm{A}$, está entre $9 \%$ e $41 \%$, o que sugere fortemente que a droga $B$ é superior à droga A na redução de vômitos no período pós-operatório.

No trabalho de Jung ${ }^{14}$, foram estudadas 161 pacientes do sexo feminino, estado físico ASA I, submetidas à cirurgia gi- 
necológica ambulatorial. As pacientes foram alocadas aleatoriamente para receber um entre quatro tratamentos: placebo, droperidol $(0,625 \mathrm{mg})$, droperidol $(1,25 \mathrm{mg})$ ou ondansetron (4 mg). O estudo em questão também é duplamente encoberto, ou seja, os pacientes e os pesquisadores não sabiam qual droga cada paciente estava recebendo. Os pacientes foram analisados dentro dos grupos para os quais foram alocados inicialmente. Com exceção da intervenção investigada, os pacientes foram tratados de forma semelhante durante o estudo. Valores de $p<0,05$ foram considerados estatisticamente significantes. Aincidência de vômitos e a necessidade de antieméticos de resgate foi significativamente menor nos grupos alocados para receber ondansetron e droperidol em comparação ao grupo placebo $(p<0,05)$, mas não diferiu entre os grupos droperidol $(0,625 \mathrm{mg} \mathrm{e} 1,25 \mathrm{mg}) \mathrm{e}$ ondansetron.

Os Benefícios do Tratamento Compensam os Danos e os Custos?

A resposta a essa pergunta deve ser dada através do conceito do número necessário para tratar (NNT). O NNT quantifica o número de pacientes que devem ser tratados para se obter um desfecho favorável a mais ou um desfecho desfavorável a menos. O NNT leva em conta não só a redução de risco relativo, mas também o risco de base para o desfecho adverso que se propõe reduzir com aquela intervenção. Matematicamente, teremos: NNT = 1/probabilidade do desfecho adverso no grupo controle - probabilidade do desfecho adverso no grupo tratamento ou (1/Redução do Risco Absoluto (RRA). O NNT é a recíproca da redução de risco absoluto (RRA), que constitui taxa de eventos em controles menos a taxa de eventos em tratados. RRR, RRAe NNT são as várias formas de sumarizar os efeitos relativos de dois tratamentos ${ }^{15}$. Considere-se novamente a droga B com seus $25 \%$ de redução de vômitos no pós-operatório. Admita-se que haja um grupo de pacientes com alto risco para vômitos (40\%) e um com baixo risco (5\%). A redução de $25 \%$ no grupo de alto risco representa uma redução de risco absoluto de $40 \%$ para $30 \%$, ou seja $10 \%$. Portanto, o NNT será $(1 / 10) \times 100=10$. Isso significa que, no grupo de alto risco, será necessário tratar 10 pacientes com a droga B para prevenir um episódio de vômito no pós-operatório. No grupo de baixo risco para vômitos, o risco absoluto diminui de $5 \%$ para $3,75 \%$, uma diferença de $1,25 \%$. O NNT é $(1 / 1,25) \times 100=80$. Portanto, no grupo de baixo risco, é necessário tratar 80 pacientes para prevenir um episódio de vômito. Antes de iniciar um tratamento, o médico deve considerar a risco do paciente para o evento adverso, pois de forma geral quanto maior for o risco de base, maiores serão os benefícios conferidos pelo tratamento.

Os Resultados do Estudo irão Melhorar o Manuseio com meus Pacientes?

Para responder a essa questão, deve-se fazer outra pergunta: qual a similaridade do meu paciente aos pacientes incluídos no estudo? Os estudos clínicos constituem experimen- tos controlados, com critérios rígidos de inclusão e exclusão. Estudos que envolvem tratamento determinam a eficácia de uma intervenção. A eficácia significa que o tratamento reduz a probabilidade de haver o efeito adverso, estritamente nas condições do estudo. É preciso determinar a efetividade do tratamento, que se refere a como o tratamento funciona na prática. Para isso, o médico deve considerar os critérios de inclusão e de exclusão e se perguntar se haveria uma razão pela qual os resultados do estudo não devessem ser aplicados ao seu paciente, ou seja, o seu paciente apresenta características muito distintas dos pacientes que participaram do estudo.

Todos os Desfechos Clínicos Importantes foram Considerados?

O tratamento deve ter impacto sobre os desfechos, que são os eventos clínicos de maior interesse para o paciente e para o médico que o assiste. Os desfechos clínicos são basicamente cinco: desenlace (morte), doença (sintomas, sinais físicos), desconforto (dor, náusea, dispnéia), deficiência funcional (limitação na capacidade de realizar atividades usuais), descontentamento (reação emocional à doença ou a seu cuidado) ${ }^{15}$. São exemplos de desfechos clínicos: redução de infarto do miocárdio, evitar hospitalização por insuficiência cardíaca congestiva ou reduzir a falta de ar para atividades diárias.

Uma alternativa ao desfecho clínico é o desfecho substituto, que constitui uma medida ou um sinal clínico utilizado em substituição ao desfecho clínico ${ }^{15}$. Ao primeiro a vantagem de utilizar o desfecho substituto é que menos pacientes podem ser envolvidos, devido ao fato de que tais desfechos geralmente são selecionados por serem mais comuns e fáceis de serem observados. Ou seja, o desfecho substituto reduzo custo e a duração do estudo.

A base para o uso do desfecho substituto é que as alterações produzidas pelo tratamento no desfecho substituto devem se refletir em alterações no desfecho clínico. Contudo, isso freqüentemente não acontece, o que tem-se verificado em estudos clínicos, como o Cardiac Arrhythmia Supression Trial, que avaliou o uso de antidisrítmicos após infarto agudo do miocárdio ${ }^{16}$. Em estudos iniciais, verificou-se que as drogas encainida e flecainida diminuem a incidência de contrações ventriculares prematuras após infarto do miocárdio. Pensava-se que deveriam diminuir também a ocorrência de disritmias graves que levam a risco de morte. Contudo, observou-se que os pacientes que recebiam essas drogas apresentavam maior mortalidade que os pacientes que recebiam placebo.

Os desfechos biológicos não podem ser substitutos adequados dos desfechos clínicos sem uma evidência direta de que os dois estejam relacionados entre si. O uso de desfechos substitutos em Anestesiologia constitui um problema em particular ${ }^{17}$. Atualmente, a mortalidade como resultado direto da anestesia é bastante reduzida. Poucos desfechos são atribuídos diretamente à anestesia. Além disso, a Anestesiologia constitui uma especialidade em particular no sentido de 
que a maioria das intervenções não são propriamente terapêuticas, mas sim destinadas a facilitar outros procedimentos. Como a incidência de desfechos adversos sérios causados pela anestesia é baixo, muitas pesquisas da área utilizam desfechos substitutos. Contudo, estes últimos não devem definitivamente substituir os desfechos clínicos. Os desfechos clínicos em Anestesiologia têm aumentado na medida em que os anestesiologistas se tornam médicos que atuam em todo o período peri-operatório. Desfechos econômicos, satisfação do paciente e melhoria de qualidade de vida relacionada à saúde também têm sido utilizados como desfechos em estudos clínicos em Anestesiologia.

\section{ESTUDOS DE DIAGNÓSTICO}

Três perguntas importantes devem ser realizadas ${ }^{18}:$ (1) Os resultados do estudo são válidos? (2) Quais são os resultados do estudo? (3) Os resultados do estudo ajudar-me-ão a cuidar dos meus pacientes?

\section{Os Resultados do Estudo são Válidos?}

\section{Guias Primários:}

(1) Foi realizada uma comparação independente e encoberta entre o teste diagnóstico proposto e o teste de referência de uso consagrado?

(2) A amostra de pacientes estudados inclui um espectro de pacientes aos quais o exame diagnóstico será aplicado na prática clínica?

\section{Guias Secundários:}

(1) Os resultados do teste avaliado influenciaram a decisão de realizar o teste padrão de referência? Um teste fraco pode ser comparado a outro teste padrão fraco, considerado como padrão de validade por ter maior uso ou por consenso de peritos. Se um teste novo for comparado a um padrão antigo, mas não exato, o teste novo poderá parecer pior, mesmo sendo melhor. Se o teste novo for mais sensível que o teste padrão, os pacientes novos identificados adicionalmente pelo teste novo serão considerados falso-positivos em relação ao teste antigo. Um exemplo clássico desse problema é o que aconteceu no trabalho de Bartrum e col. ${ }^{19}$, que compararam a ultra-sonografia dinâmica à colecistografia oral para detecção de cálculos biliares. A ultra-sonografia foi positiva em cinco pacientes, nos quais não foram detectados cálculos a partir de colecistogramas orais. Posteriormente, dois desses pacientes foram operados e os cálculos biliares encontrados, mostrando que o teste de referência foi menos exato que o novo;

2) Os métodos utilizados para realizar o teste diagnóstico foram descritos em detalhe suficiente para permitir replicação? Quando os autores chegam à conclusão como se deve usar um determinado método diagnóstico, eles obrigatoriamente devem dizer como realizá-lo.

\section{Quais são os Resultados do Estudo?}

Uma vez considerados os resultados de estudo válidos, o próximo passo é determinar a precisão do teste diagnóstico. Para isso, deve-se conhecer os conceitos de sensibilidade, especificidade, valores preditivos positivo e negativo e razão de verossimilhança (likelihood ratio), que são as principais medidas obtidas nos estudos sobre diagnóstico.

Sensibilidade consiste na proporção dos indivíduos com a doença que apresentam um teste positivo. O teste sensível é o de escolha quando a penalidade por deixar de diagnosticar uma doença é grande, por exemplo nos casos de doenças graves ou transmissíveis como tuberculose, sífilis ou de neoplasias malignas. Também são testes úteis para reduzir o grande número de possibilidades diagnósticas nas fases iniciais de uma investigação ${ }^{15}$.

Especificidade constitui a proporção dos indivíduos sem a doença que têm um teste negativo. Um teste específico raramente classificará erroneamente pessoas sadias em doentes, sendo útil para confirmar um diagnóstico sugerido por outros dados. Um teste altamente específico é raramente positivo na ausência de doença, ou seja, dá poucos resultados falso-positivos, sendo interessante quando estes resultados puderem lesar o paciente física, emocional ou financeiramente. A sensibilidade e a especificidade constituem propriedades de um teste levadas em conta ao decidir sobre a solicitação de um exame. Uma vez com o resultado na mão, a sensibilidade e e especificidade têm menos relevância. A partir desse ponto, deve-se determinar se o paciente tem ou não a doença, dados os resultados do teste. A probabilidade de doença, dados os resultados de um teste, é chamada de valor preditivo do teste. $O$ valor preditivo positivo de um teste é a probabilidade de doença em um paciente com resultado positivo. $\mathrm{O}$ valor preditivo negativo é a probabilidade de não ter a doença quando o resultado é negativo (normal).

O valor preditivo de um teste não é propriedade apenas do teste; depende da prevalência da doença na população estudada, sendo prevalência a proporção de pessoas com a condição em questão, em uma população definida, em um determinado momento.

Outro conceito importante é o da razão de verossimilhança (razão de probabilidades ou likelihood ratio) para determinado valor de um teste diagnóstico, definida como a probabilidade de tal resultado em pessoas com a doença, dividida pela probabilidade do mesmo resultado em pessoas sem a doença. As razões de probabilidades expressam quantas vezes mais (ou menos) provável é encontrar um resultado de um teste em pessoas doentes comparadas com as não doentes.

Os Resultados Obtidos Auxiliarão a Melhorar o Cuidado ao meu Paciente?

O terceiro passo consiste em decidir se o uso do teste é aplicável a um indivíduo em especial e aos pacientes da prática diária. São perguntas que devem ser feitas: os resultados do estudo são generalizáveis, ou seja, o médico pode aplicá-los aos pacientes que atende com maior freqüência na sua práti- 
ca diária? O teste diagnóstico em questão fornece informações adicionais àquelas obtidas pela história e pelo exame físico? O novo teste proposto é mais barato e mais acessível do que outros testes disponíveis para o mesmo propósito?

\section{ESTUDOS DE PROGNÓSTICO}

Prognóstico refere-se aos possíveis desfechos de uma doença e à freqüência com a qual eles possam ocorrer. Freqüentemente, as características de um paciente em particular podem ser utilizadas para predizer mais adequadamente o desfecho em questão. Essas características são chamadas fatores prognósticos. Estes podem ser de vários tipos: demográficos (p. ex.: idade, sexo), específicos da doença ( $p$. ex.: estágio do tumor) ou comorbidade (p. ex.: outras condições acompanhando a doença em questão).

Usualmente, é antiético agir de forma aleatória com pacientes para que sejam expostos a diferentes fatores prognósticos. Dessa forma, o melhor desenho de estudo para identificar a presença de riscos e determinar seu aumento associado a um fator prognóstico é o estudo de coorte. Nesse estudo, um ou mais grupos de indivíduos que ainda não sofreram o evento adverso, coortes, são seguidos por um intervalo de tempo em que ocorrem os eventos nos grupos. A partir destes estudos é possível comparar a taxa de eventos na coorte exposta ao fator em questão com a taxa de eventos na coorte não exposta e obter estimativas de risco, como o risco relativo (taxa de eventos entre os expostos/taxa de eventos entre os não-expostos). O estudo de coorte ideal é aquele que consiste numa amostra representativa da população geral e que utiliza critérios objetivos para definição dos desfechos.

Outra forma de estudar fatores prognósticos é comparar grupos de indivíduos que já apresentaram o desfecho clínico com um grupo "controle" que ainda não sofreu. Neste tipo de estudo (caso-controle), os investigadores contam o número de indivíduos em cada grupo com um fator prognóstico particular. O potencial para vieses de amostragem, bem como a natureza retrospectiva da coleta de dados, limita o grau de inferência que os clínicos podem obter desses estudos. Entretanto, os estudos de caso-controle freqüentemente são a única forma viável de responder à questões sobre etiologia de doenças, especialmente porque facilitam o estudo de doenças de ocorrência rara.

Estudos de coorte e de caso-controle são estudos de observação quanto a fatores de risco.

Três perguntas principais devem ser feitas para a interpretação de estudos de prognóstico: (1) Os resultados do estudo são válidos? (2) Quais foram os resultados? (3) Os resultados obtidos ajudar-me-ão a melhorar o cuidado aos meus pacientes? ${ }^{20}$.

\section{Os Resultados do Estudo são Válidos?}

\section{Guias Primários:}

1) Havia uma amostra representativa e bem definida de pacientes em ponto similar do curso da doença? Muitos vieses relacionados à seleção dos pacientes podem distorcer os resultados do estudo. Os autores devem descrever o estágio da doença em que os pacientes se entravam no estudo;

2) O seguimento dos pacientes foi suficientemente longo e completo? Quanto menor o número de pacientes disponíveis para seguimento, menor a acurácia para estimar o risco de um desfecho adverso. É importante considerar a relação entre a proporção de pacientes que não estão disponíveis para seguimento e os pacientes que sofreram o desfecho de interesse. Quanto maior o número de pacientes cujo destino é desconhecido em relação ao número que sofreu um evento, maior a ameaça à validade do estudo. Por outro lado, se as razões para desaparecimento dos pacientes não estiverem relacionadas ao desfecho clínico e eles forem similares àqueles que completaram o trabalho, o leitor poderá ter maior segurança quanto à validade dos resultados do estudo.

\section{Guias Secundários:}

1) Foram utilizados critérios de desfecho objetivos e sem vieses? Os investigadores devem fornecer uma definição clara e cientificamente adequada dos desfechos adversos antes do início do estudo. Para reduzir viés, o indivíduo que define o desfecho não deve ter acesso a potenciais fatores prognósticos dos pacientes;

2) Foi realizado ajuste para fatores prognósticos importantes? Ao comparar o prognóstico de dois grupos de pacientes, os investigadores devem verificar se suas características clínicas são similares e ajustar as análises para as diferenças encontradas.

\section{Quais foram os Resultados?}

Os resultados quantitativos dos estudos de prognóstico ou de risco são o número de eventos que ocorrem num determinado período.

Torna-se importante descrever as principais medidas de efeito obtidas nos estudos sobre etiologia e prognóstico: risco relativo, obtido nos estudos de coorte e razão de chances (odds-ratio), obtido nos estudos caso-controle.

Em um estudo de coorte, a população susceptível é dividida, no início, em dois grupos: expostos a um determinado fator e não-expostos. Como se pode decidir se há um aumento de risco? Deve-se calcular o Risco Relativo, que corresponde à incidência da doença nos expostos dividida pela incidência da doença nos não-expostos.

Os estudos de caso-controle começam com a seleção de um grupo que apresenta a doença e outro de controles. Não há como saber a incidência da doença porque os grupos são determinados pelo critério de seleção dos investigadores. Portanto, não é possível obter o risco relativo. Nesse caso, pode-se calcular as freqüências relativas de pessoas expostas ao fator em questão nos casos e nos controles. A comparação de exposição entre casos e controles fornece uma medida de risco semelhante conceitual e matematicamente ao risco relativo, a razão de chances (odds ratio). A razão de chances, também denominada razão de improbabilidades, é 
definida como as chances de que um caso seja exposto, dividida pelas chances de que um controle seja exposto. Se a freqüência de exposição for maior entre os casos, a razão de chances será maior que 1 , indicando aumento no risco. Assim, quanto mais intensa for a associação entre a exposição e a doença, maior será a razão de chances. Portanto, o significado da razão de chances é análogo ao do risco relativo. Contudo, a razão de chances é aproximadamente igual ao risco relativo apenas quando a incidência da doença é baixa. Assim, como já citado, os estudos de caso-controle são indicados para o estudo de doenças raras.

\section{Os Resultados Ajudar-me-ão a Cuidar do meu Paciente?}

Três perguntas devem serfeitas: (1) Os pacientes estudados são semelhantes ao meu paciente? Os autores devem descrever os pacientes do estudo detalhadamente, o suficiente para permitir a comparação com os seus pacientes; (2) Os resultados levar-me-ão a selecionar ou excluir uma terapia? Dados de prognóstico provêm freqüentemente base para decisão sobre terapia. Saber o provável curso clínico da doença do seu paciente pode ajudar a decidir se um determinado tratamento deve ou não ser utilizado; (3) Os resultados são úteis para confortar ou aconselhar pacientes.

\section{A PRÁTICA DA MEDICINA BASEADA EM EVIDÊNCIAS MELHORA O CUIDADO AO PACIENTE?}

A literatura emergente sugere que a prática da Medicina baseada em evidências melhora o cuidado com o paciente. Nos últimos quarenta anos, os conhecimentos provenientes de estudos clínicos aleatórios têm sido a base para evolução dos conceitos sobre terapia em Medicina ${ }^{21,22}$. Não há evidências provenientes de estudos clínicos aleatórios de que a prática da Medicina baseada em evidências esteja associada a melhores desfechos clínicos, mas há evidências provenientes de estudos de efetividade de que os pacientes que recebem terapia baseada em evidências possuem melhores desfechos clínicos que os que não recebem e de que os residentes treinados de acordo com os princípios da Medicina baseada em evidências mais freqüentemente utilizam estes princípios e tratamentos adequados ${ }^{23}$.

Além disso, o uso de sistemas computadorizados de tomada de decisão em Medicina, com forte embasamento em evidências científicas atualizadas, podem mudar o comportamento do médico e melhorar o manuseio com o paciente ${ }^{24,25}$.

\section{CONCLUSÃO}

A Medicina baseada em evidências constitui um novo paradigma que enfatiza os raciocínios crítico e investigativo. Apesar de inicialmente relacionada à Medicina Interna, a prática da Medicina baseada em evidências foi incorporada à Anestesiologia e sua integração à prática diária do anestesiologista pode melhorar o manuseio com os pacientes.

\section{Evidence-Based Anesthesiology: What Is It and How to Practice It}

Rodrigo Mariano da Costa de Angelis, TSA, M.D.; Álvaro Avezum Júnior, M.D.; Alexandre Biasi Cavalcanti, M.D.; Rogério Teixeira de Carvalho, M.D.

\section{INTRODUCTION}

Evidence-based medicine, expression used for the first time in Brazil by Avezum ${ }^{1}$, the origin of which is France, $19^{\text {th }}$ Century, is the conscious, explicit and judicious use of the best evidence aiming at decision making for the treatment of individual patients ${ }^{2}$. Major evidence-based medicine principle is the effective use of medical literature as a guide for daily practice 3 . There are three key-elements: best scientific evidence, physicians' clinical experience and patients' preferences.

The expression evidence-based medicine was introduced in the medical literature approximately 20 years ago ${ }^{4}$, and in spite of being initially related to Internal Medicine, it has been incorporated to Anesthesiology. The first textbook on evidence-based Anesthesiology was published in 2000, in the UK ${ }^{5}$.

The last decade has witnessed an increasing interest on the subject, attributed by Sackett et al. ${ }^{6}$ to four basic reasons: the increasing need for knowledge about diagnosis, prognosis, therapy and prevention; limitations of traditional information sources, such as textbooks (often outdated), specialists (very often giving inaccurate opinions based on inadequate scientific grounds) and continued medical education (most not so effective); disparity between physicians' diagnostic ability and clinical reasoning, which tend to improve with age and experience, and their scientific knowledge and clinical performance, which tend to decrease with age and lack of time for studying, very often resulting in only 30 minutes/week dedicated to searching and assimilating knowledge ${ }^{4}$.

It is estimated that 3 hours and 19 minutes are needed every day for physicians to remain updated on their specialty. Societies of Anesthesiology have tried to apply evidence-based Medicine principles to improve patients' care. As result, anesthesiologists are considered pioneers in the development and implementation of guidelines, which seem to have contributed to the sustained and disseminated decrease in anesthesia-related mortality and morbidity ${ }^{7}$. However it is difficult to establish a strong causal relationship between the activities of those Societies and the decrease in morbidity and mortality.

\section{PRACTICE OF EVIDENCE-BASED MEDICINE}

The practice of evidence-based medicine implies the integration of individual clinical experience and best available external evidences produced by clinical research ${ }^{8}$, and consists of five basic steps: translate information needs into a well structured clinical question; look for the best evidence to answer the question; evaluate the evidence in terms of validity and 
applicability to the clinical practice; integrate the best evidence to personal experience and patients' preferences to put it into practice; and evaluate the effectiveness of measures adopted in search for continuous improvement ${ }^{9}$.

How can evidence-based medicine be applied to Anesthesiology? It may be incorporated to our daily practice and also to review meetings, case discussions and teaching of the specialty. Anesthesiologists may replace review meetings and case discussions based on non-systematized reviews, by systematized reviews with clinical cases and literature evaluation.

\section{CLINICAL QUESTION FORMULATION}

A well-structured clinical question is the first step for decision making in evidence-based medicine. Agood clinical question should be strictly relevant to the problem and asked in a way to help the search for the answer. Well-structured questions have four key-elements: (1) the patient or problem of interest; (2) major intervention, that is, exposure, diagnostic test, prognostic factor or treatment regimen; (3) comparison between exposure interventions, if applicable; (4) clinical outcome of interest ${ }^{6}$.

Questions related to therapy, diagnosis and prognosis are of special interest for anesthesiologists. Some examples of clinical Anesthesiology questions are: should patients submitted to total hip replacement receive general or regional anesthesia? Which is the best strategy to prevent postoperative vomiting? Which is the probability of one patient with restrictive pulmonary disease, whose preoperative vital capacity is $30 \%$ of normal, need postoperative mechanical ventilation? For patients who, in previous exposure to anesthesia, there has been suspicion of malignant hyperthermia, what is the ability of the in vitro contracture test to predict who will develop the problem? Would low molecular weight heparin for a female patient aged 80 years submitted to hip surgery improve her prognosis? Which is the risk for bronchospasm in a patient receiving rapacuronium as neuromuscular blocker? 10,11

After developing a well-structured question the next step is to look for the best possible evidence.

\section{SEARCH FOR THE BEST EVIDENCE}

There are basically four ways to obtain information: ask somebody, check a textbook, find an article related to the subject in your personal files or use a database. Asking a professor or a colleague is a highly effective way; however it only makes sense when it is a rare situation or case which the professional will seldom face again. Check a textbook may be interesting, although they are in general outdated as from the moment of their publication. Refer to a personal file article implies the risk that data were not maintained updated and complete.

Currently, electronic databases are the fastest and more updated means to find information. Largest and most widely used data source is MedLine, which counts on more than
3500 indexed journals with approximately 11 million references from 1966 to date. Medical subject heading, $\mathrm{MeSH}$, is the name of the standard vocabulary used to index MedLine references. The hierarchic database organization follows a branched structure which allows the user to use more specific or more generic terms to change the strategy of the search. $\mathrm{MeSH}$ query allows the search by subject, providing a better result as compared to searching just by words present on the text. MedLine may be accessed by the PubMed of the National Library of Medicine, by Ovid Technologies, MdConsult and other electronic information sources.

Specifically for Anesthesiology, information is spread throughout general medicine journals of multiple sub-specialties, including Anesthesiology itself, Pediatrics and Surgery, in addition to basic sciences.

The search for MedLine answers should be based on the four already mentioned clinical question elements.

MedLine provides access to most published biomedical literature. However, it does not critically evaluate database references. Obtained information analysis is the next step for the practice of evidence-based medicine. Physicians who read a lot but do not evaluate the validity and applicability of the source are well informed, but have not learned and will not have adequate subsidies to argue in favor of their management.

\section{STUDIES ANALYSIS}

\section{Treatment Studies}

Five questions have to be asked for treatment studies ${ }^{12,13}$ : (1) are study results valid? (2) Which were the outcomes? (3) Have treatment benefits overcome damages and costs? (4) Will outcomes help improve my patients' care? (5) Were all major clinical outcomes taken into consideration?

\section{Are Study Results Valid?}

First we have to investigate whether the study represents an unbiased estimate of the effects of the approach or if there is some type of bias, that is, systematic error in an epidemiologic study resulting in incorrect estimate of the association between exposure and outcome. This question may be answered by the following questions: (1) Were patients randomly selected for each treatment group? Random distribution decreases biases and assures that clinical outcomes determinants are homogeneously distributed among groups; (2) Were all selected patients computed? Have all patients starting the study finished it? Were patients who have not concluded the study included in the final analysis? (3) Were patients analyzed according to the groups they were randomly allocated to in the beginning of the study (treating intention principle)? (4) Is it a double-blind study? In a double-blind study, patients and investigators do not know the treatment option; (5) With the exception of the intervention being investigated, were patients treated equally throughout the study? This latter question is closely related to the previ- 
ous one: in open studies, in which physicians and patients know the treatment being administered, both patients' behavior after knowing the actual treatment they are receiving and the way clinicians manage the case may be modified. End result will be that study results will not reflect just the effects of the treatment being tested, but also the effects of other treatments which are different among groups. Adopting the double-blind scheme may prevent this problem.

An example of this type of study related to Anesthesiology is a randomized double-blind study comparing drugs $A$ and $B$ as to their efficacy in preventing perioperative vomiting, such as the study performed by Tang et al., who have compared the efficacy of ondansetron and droperidol to control perioperative vomiting of elective gynecological surgeries ${ }^{14}$.

\section{Which were the Results?}

Two questions should be asked with regard to results: (1) Which has been the magnitude of observed therapeutic effect? (2) What is the accuracy of therapeutic effect estimate? Consider that in the above-mentioned study about perioperative vomiting, the incidence of nausea and vomiting in the group of patients allocated to drug A has been $20 \%$ and that in group receiving drug $B$ this has been observed in $15 \%$ of cases. The risk for perioperative nausea and vomiting in group $B$ as compared to group $A$ is $0.15 / 0.20=0.75$. This is called relative risk $(R R)$. Relative risk is the ratio of events rate in the treated group divided by events rate in control group (TER/CER). Another important concept is relative risk reduction (RRR) which is control group events rate less treated group events rate divided by control group events rate (CER - TER)/CER or (1 - RR) x 100. RRR is expressed in percentage. The new therapy (drug $B$ ) has decreased the risk for nausea and vomiting in $25 \%$ as compared to conventional therapy (drug A). But which is the accuracy of this $25 \%$ decrease? This depends on the number of patients enrolled in the study, that is, sample size. Let's say that the study included 100 patients; there would be 20 vomiting episodes in group $A$ and 15 in group $B$, a difference of just 5 episodes. If the number of patients increased to 1000 , there would be 200 episodes in group $A$ and 150 in group $B$, a difference of 50 episodes. RRR is the same, but confidence interval will be narrower, indicating a higher accuracy of results. This happens because the higher number of events decreases the possibility of results having been reached by chance.

The confidence interval informs values within which the true effect should be located. It combines information about the strength of an association (such as relative risk reduction) with information about effects of chance on the observed result.

By convention, confidence interval used is $95 \%$, meaning that there is $95 \%$ probability that actual population value is within the limit found. The larger the sample size, the higher will be the number of events observed and more convinced readers may be that the result is a true and more accurate representation of values observed in the general population.
Confidence intervals interpretation is relatively simple. In our previous example there are two situations. The study involving 100 patients had a confidence interval for RRR of $-38 \%$ (increased incidence of nausea and vomiting with drug $B$ ) to $59 \%$ (decreased incidence of vomiting with drug B). Since $95 \%$ confidence interval includes zero, it is considered that the studied effect is not statistically significant. In the study involving 1000 patients, confidence interval was $9 \%$ to $41 \%$, meaning that readers may be $95 \%$ sure that vomiting decrease with drug $B$ is between $9 \%$ and $41 \%$, strongly suggesting that drug $B$ is better than drug A to decrease perioperative vomiting.

Jung ${ }^{14}$ has studied 161 female patients physical status ASAI, submitted to outpatient gynecologic surgeries. Patients were randomly allocated to receive one out of four regimens: placebo, droperidol (0.625 mg), droperidol $(1.25 \mathrm{mg}$ ) or ondansetron ( $4 \mathrm{mg}$ ). This study was also double blind, that is, patients and investigators were blind to the drug each patient was receiving. Patients were evaluated within the groups they were initially allocated to. With the exception of the investigated intervention, patients were similarly treated throughout the study and $p<0.05$ was considered statistically significant. The incidence of vomiting and the need for rescue anti-emetics has been significantly lower in the ondansetron and droperidol groups as compared to placebo $(p<0.005)$, but has not differed between droperidol $(0.625 \mathrm{mg}$ and 1.25 $\mathrm{mg}$ ) and ondansetron groups.

\section{Have Treatment Benefits overcome Damages and Costs?}

This question should be answered through the concept of the number needed to treat (NNT). NNT quantifies the number of patients to be treated to obtain one additional favorable outcome or one unfavorable outcome less. NNT takes into account not only relative risk reduction but also the baseline risk for the adverse outcome such intervention is attempting to decrease. In mathematical terms, we have: NNT = 1/probability of adverse outcomes in the control group - probability of adverse outcome in the treatment group or (1/Absolute Risk Reduction (ARR). NNT is the reciprocate of absolute risk reduction (ARR), which is the events rate in controls less events rate in treated. RRR, ARR and NNT are different ways to summarize relative effects of two treatments ${ }^{15}$. Let's once more consider drug B with its $25 \%$ perioperative vomiting decrease. Let's admit that there is a group of patients at high risk for vomiting $(40 \%)$ and one at low risk $(5 \%)$. The decrease of $25 \%$ in the high risk group represents absolute risk decrease from $40 \%$ to $30 \%$, that is, $10 \%$. NNT will be $(1 / 10) \times 100=10$. This means that 10 patients will have to be treated with drug $B$ in the high risk group to prevent one postoperative vomiting episode. In the low risk group, absolute risk decreases from $5 \%$ to $3.75 \%$, or a difference of $1.25 \%$. NNT is $(1 / 1.25) \times 100=$ 80 . So, it is necessary to treat 80 low risk patients to prevent one vomiting episode. Before starting a treatment, physicians should consider patients' risk for the adverse event, because in general, the higher the baseline risk, the higher will be the benefits of the treatment. 
Will outcomes Help Improve my Patients' Care?

This question could be answered by another question: which is the similarity of my patient and patients included in the study? Clinical trials are controlled experiments, with strict inclusion and exclusion criteria. Studies involving treatments determine the efficacy of an intervention. Efficacy means that the treatment decreases the probability of an adverse effect, strictly within study conditions. It is necessary to determine the effectiveness of the treatment, or how does the treatment work in practice. For such, physicians should consider inclusion and exclusion criteria and ask themselves whether there is a reason why study results should not be applied to their patients, that is, patients are much different than patients participating in the study.

\section{Were all Major Outcomes taken into Consideration?}

Treatment should impact outcomes, which are clinical events most interesting patients and physicians. There are basically 5 clinical outcomes: end (death), disease (symptoms, physical signs), distress (pain, nausea, dyspnea), functional impairment (limited ability to perform usual activities), unhappiness (emotional reaction to disease or care) ${ }^{15}$. Examples of clinical outcomes are: decreased myocardial infarction, prevent admission for congestive heart failure or decrease dyspnea of air for daily activities.

One alternative to clinical outcome is substitute outcome, which is a measure or clinical sign used to replace clinical outcome ${ }^{15}$. The advantage of the substitute outcome is that fewer patients may be involved due to the fact that such outcomes are in general selected for being more common and easy to be observed. That is, the substitute outcome decreases study costs and duration.

The basis for the substitute outcome is that changes on substitute outcome promoted by treatment should be reflected in changes in clinical outcome. However, this is often not true and is what has been seen in studies such as Cardiac Arrhythmia Suppression Trial, which has evaluated the use of anti-arrhythmics after acute myocardial infarction ${ }^{16}$. In preliminary studies, it has been observed that encainide and flecainide decrease the incidence of early ventricular contractions after myocardial infarction. It has been thought that they would also decrease the incidence of life-threatening severe arrhythmias. However, it has been observed that patients receiving such drugs had higher mortality rates as compared to patients receiving placebo.

Biological outcomes cannot be adequate substitutes for clinical outcomes without direct evidence that both are inter-related. The use of substitute outcomes in Anesthesiology is a special problem ${ }^{17}$. Currently, mortality as direct result of anesthesia is highly decreased. Few outcomes are directly attributed to anesthesia. In addition, Anesthesiology is a unique specialty in the sense that most interventions are not exactly therapeutic, but aimed at helping other procedures. Since the incidence of anesthesia-induced severe adverse events is low, many studies in the area use substitute out- comes. However, the latter should not definitively replace clinical outcomes. Clinical outcomes in Anesthesiology have increased, as anesthesiologists become physicians acting throughout the perioperative period. Economic outcomes, patients' satisfaction and improved quality of life have also been used as outcomes in clinical trials in Anesthesiology.

\section{DIAGNOSTIC STUDIES}

Three important questions should be asked ${ }^{18}$ : (1) Are study results valid? (2) Which were the study results? (3) Will results help me improve patients' care?

\section{Are Study Results Valid?}

Primary Guidelines:

(1) Was an independent and blind comparison performed between the proposed diagnostic test and the widely used reference test?

(2) Does sample size include a spectrum of patients in whom the diagnostic test will be applied in the clinical practice?

Secondary Guidelines:

(1) Have test results influenced the decision of performing the standard reference test? A weak test may be compared to other weak standard test considered the validity standard for being widely used or consensus among experts. If a new test is compared to an old but inaccurate test, the new test may seem worse even if being better. If the new test is more sensitive than the standard test, new patients additionally identified by the new test will be considered false-positives as compared to the old test. A classic example is what has happened in the study by Bartrum et al. ${ }^{19}$ who have compared dynamic ultrasound and oral cholecystography to detect billiary stones. Ultrasound was positive in 5 patients in whom no stones were detected by oral cholecystographies. Afterwards, two of these patients were operated on and stones were found, showing that the reference test was less accurate than the new test;

(2) Were methods used for diagnostic test described in enough detail to allow replication? When authors reach a conclusion on how to use a certain diagnostic test, they are to explain how to perform it.

\section{Which were Study Results?}

Once study results are considered valid, next step is to establish diagnostic test accuracy. For such, the concepts of sensitivity, specificity, positive and negative predictive values and likelihood ratio should be understood, because they are major measurements obtained by diagnostic studies.

Sensitivity is the percentage of individuals with the disease with a positive test. A sensitive test is chosen when there is major penalty for not diagnosing a disease, such as severe or transmissible diseases like tuberculosis, syphilis or malig- 
nant neoplasias. These are also useful tests to decrease the large number of diagnostic possibilities during early phases of an investigation ${ }^{15}$.

Specificity is the percentage of individuals without the disease with a negative test. A specific test will seldom misclassify healthy people as sick people and is useful to confirm diagnosis suggested by other data. A highly specific test is seldom positive in the absence of disease, that is, gives few false-positive results, being of interest when such results may physically, emotionally or financially hurt the patient. Sensitivity and specificity are tests properties taken into account when deciding about asking for a test. With results in hands, sensitivity and specificity are not so relevant. As from this point, it has to be determined whether the patient has or not the disease given tests results. The probability of disease given test results is called test predictive value. Positive predictive value is the probability of disease in a patient with positive result. Negative predictive value is the probability of not having the disease when results are negative (normal).

The predictive value of a test is not a property unique to the test; it depends on the prevalence of the disease among studied population, being prevalence the number of people with the studied condition in a defined population in a certain moment.

Another important concept is likelihood ratio (odds ratio) for a certain value of a diagnostic test, defined as the probability of such result in people with the disease, divided by the probability of the same result in healthy people. Likelihood ratio expresses how many times more (or less) likely it is to find a test result in sick people as compared to healthy people.

\section{Will Results help Improving my Patients' Care?}

The third step is to decide whether the test is applicable to a specific individual and to daily practice patients. Questions to be asked are: may study results be generalized, that is, can the physician apply them to patients most frequently seen in his/her daily practice? Does this diagnostic test supply additional information to the history and physical evaluation? Is the new proposed test cheaper and more accessible than other available tests for the same aim?

\section{PROGNOSTIC STUDIES}

Prognostic refers to possible outcomes of a disease and the frequency in which they may occur. Often, the characteristics of a certain patient may be used to more accurately predict the outcome. These characteristics are called prognostic factors and may be of several types: demographic (e.g., age, gender), specific of the disease (e.g. tumor stage) or co-morbidity (e.g., other conditions following the disease).

In general, it is anti-ethic to randomly act with patients for them to be exposed to different prognostic factors. So, the best study design to identify risks and determine their increase associated to a prognostic factor is the cohort study. In this study, one or more groups of individuals who have still not suffered an adverse event, or cohorts, are followed for a time period in which events have occurred within groups. As from these studies, it is possible to compare events rate in the cohort exposed to a certain factor to events rate in the cohort not exposed, and obtain risk estimates, such as relative risk (events rate among exposed people/events rate among non-exposed people). The optimal cohort study is that consisting of a representative sample of general population using objective criteria to define outcomes.

Another way to study prognostic factors is to compare groups of individuals who have already presented the clinical outcome to a control group without clinical outcome. In this type of study (case-control), investigators count the number of individuals in each group with a certain prognostic factor. The potential for sampling biases, as well as the retrospective nature of data collection, limits the level of interference clinicians may obtain from those studies. However, case-control studies are often the only feasible way to answer questions about etiology, especially because they help the study of rare diseases.

Cohort and case-control studies are observations of risk factors. Three major questions should be asked for the interpretation of prognostic studies: (1) Are study results valid? (2) Which were the results? (3) Results obtained will help me improve patients' care?

\section{Are Study Results Valid?}

Primary Guidelines:

1) Was there a representative and well-defined sample of patients at similar stages of the disease? Many biases related to patients' selection might distort results. Authors should describe the stage of the disease in which patients entered the study;

2) Was patients' follow-up sufficiently long and complete? The smaller the number of patients available for follow up, the lower the accuracy to estimate the risk of an adverse outcome. It is important to consider the ratio between the percentage of patients not available for follow up and patients suffering an outcome of interest. The higher the number of patients with unknown outcome as compared to the number suffering an event, the higher the threat to the validity of the study. On the other hand, if the reasons for patients' disappearance is unrelated to clinical outcome and if they are similar to those completing the study, the reader may be more comfortable as to study results validity.

\section{Secondary Guidelines:}

1) Were outcome criteria objective and unbiased? Investigators should supply a clear and scientifically adequate definition of adverse outcomes before starting the study. To decrease bias, the individual defining the outcome should not have access to patients' prognostic factors;

2) Was an adjustment performed for major prognostic factors? In comparing the prognosis of two groups of patients, investigators should check whether their clinical characteristics are similar and adjust the analysis for differences found. 
Which were the Results?

Quantitative prognostic or risk studies results are the number of events occurring in a certain period.

It is important to describe major effect measures obtained in studies about etiology and prognosis: relative risk obtained in cohort studies and odds ratio obtained in case-control studies.

In a cohort study, susceptible population is initially divided in two groups: exposed and not exposed to a certain factor. How to decide whether there is increased risk? Relative Risk should be calculated and corresponds to the incidence of disease in exposed patients divided by the incidence of disease in non-exposed people.

Case-control studies start with the selection of a group with the disease and a control group. There is no way to know the incidence of the disease because groups are selected according to investigators' selection criteria. So, it is impossible to obtain the relative risk. In this case, relative frequency of people exposed to the factor in cases and controls may be calculated. The comparison of the exposure between cases and controls gives a risk measurement conceptually and mathematically similar to relative risk, which is odds ratio. Odds ratio, also called unlikelihood ratio, is defined as the chances of a case being exposed divided by the chances of a control being exposed. If exposure frequency is higher among cases, odds ratio will be higher than 1 , indicating increased risk. So, the more intense the association between exposure and disease, the higher will be the odds ratio. So, the meaning of odds ratio is similar to that of relative risk. However, odds ratio is approximately equal to relative risk only when there is low incidence of the disease. So, as already mentioned, case-control studies are indicated for the study of rare diseases.

\section{Will Results help me Improve Patients' Care?}

Three questions should be asked: (1) are studied patients similar to my patient? Authors should describe studied patients in detail, enough to allow a comparison with your patients. (2) Will results lead me to select or exclude a therapy? Prognostic data are often the basis for deciding the therapy. Knowing the potential clinical course of your patient's disease may help decide whether a treatment should or should not be used. (3) Are results useful to comfort or counsel patients?

\section{DOES EVIDENCE BASED MEDICINE IMPROVE PATIENTS' CARE?}

Emerging literature suggests that the practice of evidence-based medicine improves patients' care. In the last 40 years, knowledge provided by randomized clinical trials has been the basis for the evolution of medical therapy concepts 21,22 . There are no evidences from randomized clinical trials that evidence-based medicine is related to better clinical outcomes, but there are evidences from effectiveness studies that patients receiving evidence-based therapy have better clinical outcomes as compared to those not receiving it, and that residents trained according to evidence-based medicine principles use these principles and adequate treatments more often ${ }^{23}$.

In addition, medical decision-making computer systems, strongly based on current scientific data, may change physician's behavior and improve patients' management ${ }^{24,25}$.

\section{CONCLUSION}

Evidence-based medicine is a new paradigm emphasizing critical and investigative reasoning. Although initially related to internal Medicine, evidence-based medicine practice has been incorporated to Anesthesiology and its integration to anesthesiologists' daily practice may improve patients' management.

\section{REFERÊNCIAS - REFERENCES}

01. Avezum A - Cardiologia baseada em evidências e a avaliação crítica da literatura cardiológica: princípios de epidemiologia clínica aplicada à cardiologia. Rev Soc Cardiol Estado de São Paulo, 1996;3:241-259.

02. Logan AS - Investigation of Toronto General Practioner's Treatment of Patients with Hypertension. Toronto: Canadian Facts, 1978.

03. Schulman SR, Schardt C, Erb T - Evidence-based Medicine in Anesthesiology. Current Opinion in Anesthesiology, 2002;15: 661-668.

04. Evidence-based Medicine Working Group. Evidence-based medicine: a new approach to teaching the practice of medicine. JAMA, 1992;268:2420-2425.

05. Tramer M - Evidence-based resource in anesthesia and analgesia. London: BMJ Books, 2000.

06. Sackett DL, Straus SE, Richardson WS et al - Evidence-Based Medicine: How to Practice and Teach EBM. New York, Churchill Livingstone, 2000.

07. Gaba D, Howard SK, Fish KJ - Crisis Management in Anesthesiology. New York, Churchill Livingstone, 1994.

08. Cook DJ, Levy MM - Evidence-based medicine. A tool for enhancing critical care practice. Crit Care Clin, 1998;14:353-358.

09. Oxman AD, Sackett DL, Guyatt GH - Users guides to the medical literature: How to get started. JAMA, 1993;270:2093-2095.

10. Rajchert DM, Pasquariello CA, Watcha MF et al - Rapacuronium and the risk of bronchospasm in pediatric patients. Anesth Analg, 2002;94:488-493.

11. Schulman SR - Rapacuronium redux. Anesth Analg, 2002;94:483-484.

12. Guyatt GH, Sackett DL, Cook DJ - Users guides to the medical literature: II. How to use an article about therapy or prevention: A. Are the results of the study valid? JAMA, 1993;270: 2598-2601.

13. Guyatt GH, Sackett DL, Cook DJ - Users guides to the medical literature: II. How to use an rticle about therapy or prevention: B. What were the results and will they help me in caring for my patients? JAMA, 1994;271:59-63.

14. Tang J, Watcha MF, White PF - A comparison of costs and efficacy of ondansetron and droperidol as prophylactic antiemetic therapy for elective outpatient gynecologic procedures. Anesth Analg, 1996;83:304-313. 
15. Fletcher RH, Fletcher SW, Wagner EH - Epidemiologia Clínica: Elementos Essenciais $3^{\mathrm{a}} \mathrm{Ed}$, Porto Alegre, Artmed, 1996.

16. Cardiac Arrhythmia Suppression Trial (CAST) Investigators. Preliminary report: effect of encainide and flecainide on mortality in a randomized trial of arrhythmia suppression after myocardial infarction. N Engl J Med, 1989;321:406-412.

17. Fisher DM - Surrogate outcomes: meaningful not! Anesthesiology, 1999;90:355-356.

18. Jaeschke R, Guyatt GH, Sackett DL - Users guides to the medical literature: III. How to use an article about a diagnostic test. B. What are the results and will they help me in caring for my patients? JAMA, 1994;271:703-707.

19. Bartrum Jr RJ, Crow HC, Foote SR - Ultrasonic and radiographic cholecystography. N Engl J Med, 1977;296:538-541.

20. Laupacis A, Wells G, Richardson WS et al - Users guides to the medical literature: $\mathrm{V}$. How to use an article about prognosis. JAMA, 1994;272:243-237.

21. Yusuf $S$ - Randomised controlled trials in cardiovascular medicine: past achievements, future challenges. BMJ, 1999;319: 564-568.

22. Baum M, Houghton J - Contribution of randomised controlled trials to understanding and management of early breast cancer. BMJ, 1999;319:568-571.

23. Kitchens JM, Pfeifer MP - Teaching residents to read the medical literature: a controlled trial of a curriculum in critical appraisal/clinical epidemiology. J Gen Intern Med, 1989;4: 384-387.

24. Durieux P, Nizard R, Ravaud P et al - A clinical decision support system for prevention of venous thromboembolism: effect on physician behavior. JAMA, 2000;283:2816-2821.
25. Randolph AG, Haynes RB, Wyatt $J$ et al - Users guides to the Medical Literature: XVIII. How to use an article evaluating the clinical impact of a computer-based clinical decision support system. JAMA, 1999;282:67-74.

\section{RESUMEN}

Angelis RMC, Avezum Jr A, Cavalcanti AB, Carvalho RT - Anestesiología Fundamentada en Evidencias: lo que es y como practicar

JUSTIFICATIVA Y OBJETIVOS: Los principios de la Medicina fundamentados en evidencias han sido aplicados a la Anestesiología. La propuesta de esa revisión es abordar los fundamentos para la práctica de la Medicina fundamentados en evidencias y proporcionar ejemplos de como eses principios pueden ser incorporados a la práctica diaria. Hasta el momento de la conclusión de ese artículo, no fue encontrado ningún trabajo sobre Anestesiología fundamentados en evidencias en la literatura médica brasileña, lo que determinó su realización.

CONTENIDO: Este artículo consiste en una revisión que aborda un nuevo paradigma de la Medicina y como él puede ser aplicado a la Anestesiología.

CONCLUSIONES: La Anestesiología fundamentada en evidencias constituye la integración de la mejor evidencia científica disponible a la experiencia clínica del anestesiologista y a las expectativas del paciente, con la finalidad de la mejoría y cuidado con él. 